\title{
Thoracic Wall Siliconoma after Silicone Leaking from a Defective Breast Implant in Patient with Poland Syndrome
}

\author{
Garreffa Emanuele $^{1^{*}}$, Torresini Guido ${ }^{1}$, Quaglione Gina $^{2}$ and Brucchi Maurizio ${ }^{3}$ \\ ${ }^{1}$ Plastic and Reconstructive Surgery Outpatients' Clinic, Division of General Surgery, Department of Surgery, Ospedale Civile Mazzini, Teramo, Italy \\ ${ }^{2}$ Chief of the Division of Pathological Anatomy, Department of Services, Ospedale Civile Mazzini, Teramo, Italy \\ ${ }^{3}$ Chief of the Breast Surgery Unit, Division of General Surgery, Department of Surgery, Ospedale Civile Mazzini, Teramo, Italy
}

"Corresponding author: Emanuele Garreffa, Plastic and Reconstructive Surgery Outpatients' Clinic, Division of General Surgery, Department of Surgery, Ospedale Civile Mazzini, Piazza Italia 164100 Teramo, Italy, E-mail: emangar@live.it

Received date: July 14, 2015, Accepted date: August 20, 2015, Published date: August 25, 2015

Copyright: (ㄷ 2015 Emanuele G, et al. This is an open-access article distributed under the terms of the Creative Commons Attribution License, which permits unrestricted use, distribution, and reproduction in any medium, provided the original author and source are credited.

\section{Introduction}

Silicone breast implants are widely used in both aesthetic and reconstructive breast surgery. Within the rare complications that can appear in patients with this type of implant there is the formation of local and peripheral siliconomas, due to the diffusion of the silicone gel contained within the implant, that can appear in case of both damaged and intact implants [1,2].

The use of new generation breast implants (double lumen envelope and content of cohesive gel) has considerably lowered, but not eliminated, the risk of rupture and the possible formation of siliconomas [3].

The reported case regards a patient who, affected by Poland Syndrome, underwent breast augmentation surgery in order to correct the defect at the age of 18. A P.I.P. implant (Poly Implant Prothese, France) was positioned and, after 10 years, at the diagnostic exams revealed a nodular mass of the thoracic wall compatible with siliconoma without evident rupture of the implant.

\section{Case Report}

A 30-years-old woman affected by left Poland Syndrome underwent breast augmentation surgery in 2002 on her left breast and a "round block" mastopexy on the right to obtain symmetrisation. At that time a P.I.P. implant was used (Figure 1).

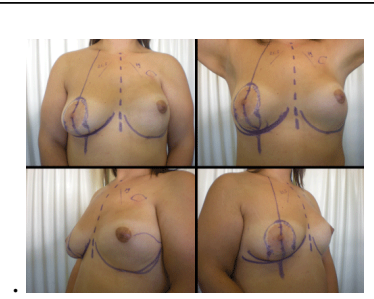

Figure 1: Pre-operative view and surgical plan, the cutaneous projection of the siliconoma is marked too.

The patient came to our office referring the appearance of a palpable nodular mass in the left inferoclavicular region. A breast MR scan and a thoracic wall TC scan were performed evidencing an area of alteration in the implant envelope without evident rupture together with the presence of a roundish area approximately $2,5 \mathrm{~cm}$ in diameter in the thoracic wall, in the left inferoclavicular region on the emiclavicular line. The mass was indicated as a possible leakingoriginated siliconoma (Figure 2).

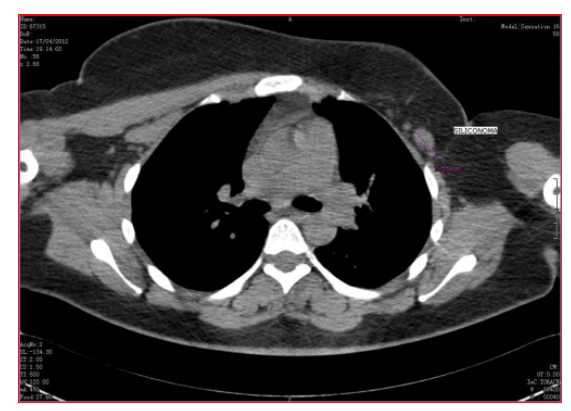

Figure 2: TC image evidencing the siliconoma

Implant replacement surgery, with contextual siliconoma removal, was scheduled.

A skin incision was performed laterally to the NAC area and, after opening the capsule a round 245 cc P.I.P. implant was removed appearing intact. After the capsulectomy we proceeded to perform a dissection trough electrocautery of the superior pole of the pocket up to the siliconoma which was isolated from the surrounding tissues and excised. After drainage was positioned, an anatomical 350 cc Mentor (Johnson \& Johnson, USA) implant was inserted in the pocket and the surgical incision was sutured. Both periprosthetic capsule and siliconoma were sent for histological testing.

On the right breast a symmetrisation vertical mastopexy was performed. Compressive medication was performed bilaterally.

On the fourth day the patient was discharged and the drainage was removed after 10 days from the surgery (Figure 3).

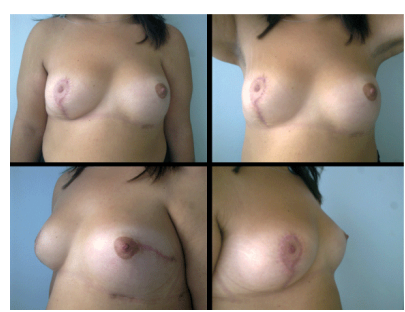

Figure 3: Clinical result 3 months after surgery 
The histological exam confirmed the diagnosis of siliconoma, specifying giant-cell histiocytic granuloma with nodular spaces filled with silicone, while the periprosthetic capsule exam evidenced synovial metaplasia on the surface in contact with the implant (Figure 4).

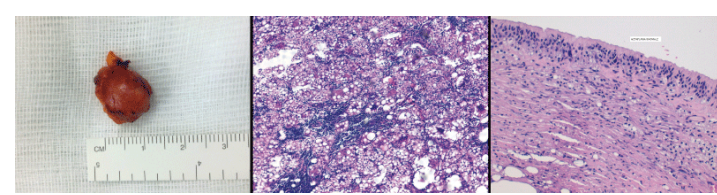

Figure 4: a) macroscopic appearance of the siliconoma. b) Histologic appearance of the siliconoma (hematoxylin and eosin). c) Histologic appearance of the periprosthetic capsule, with the presence of synovial metaplasia (hematoxylin and eosin).

\section{Discussion}

Poland Syndrome is a rare disease, with an incidence of 1/20-30.000, characterised by monolateral thoracic and/or upper-limb muscles abnormalities (aplasia/hypoplasia), associated with breast aplasia/hypoplasia, rib hypoplasia, syndactyly, and abnormalities of heart, kidneys and genitalia. In the majority of cases to correct the breast defect, after the preparation of a subcutaneous pocket, a silicone breast implant or a breast expander (subsequently replaced with an implant) is positioned [4].

In our patient's case the selected surgery was the positioning of a P.I.P. silicone breast implant.

According to investigation carried out in France, in the period from 2001 to 2010 the P.I.P. firm (Poly Implant Prothese, France), produced and exported, in 64 countries, silicone breast implants which did not conform with safety standards (greater risk of rupture and employment of a silicone gel with different components from those reported in the "technical file") [5].

In our country it is estimated that about 4.300 women have this type of implant. In fact, on February 9, 2012, the Ministry of Health arranged that all women with P.I.P. implants (positioned in both aesthetic and reconstructive surgery) could undergo replacement surgery, taking advantage of the NHS5.

This measure was introduced as a result of the elevated number of proven cases of complications in patients with implants produced by this firm, but also as a means of preventing any possible contact of tissues with the silicone contained in the implants, possessing high irritating capacities.

In patients with breast implants complications such as siliconomas and lymphadenopathy from implant failure can develop even after many years, and the only indication that is shared in the little literature published on this topic is that they must be surgically removed as soon as diagnosed [6].

In the patient that we treated the only evident localization of the silicone was on the thoracic wall, since there were no further detectable accumulations during physical exam and diagnostic imaging exams. It's noteworthy that the silicone leakage occurred through an intact implant; indicative data of the poor quality of the materials used by the P.I.P. firm to produce the prosthetic envelope, which resulted unable to contain the silicone gel.

\section{Summary}

In the period from 2001 to 2010 the P.I.P. firm (Poly Implant Prothese, France) produced breast implants not conforming to safety standards. One of this implant was used on our patient to correct Poland syndrome and, after 10 years, a thoracic wall siliconoma appeared. We provided the siliconoma excision and the implant replacement, performing at the same time a contralateral mastopexy to improve the symmetry and the overall aesthetic outcome of the reconstruction.

\section{References}

1. Puckett CL (2000) On the safety of silicone gel breast implants. Cancer Invest 18: 278-280

2. Lykissa ED, Kala SV, Hurley JB, Lebovitz RM (1997) Release of low molecular weight silicones and platinum from silicone breast implants. Anal Chem 69: 4912-4916.

3. Berg WA, Caskey CI, Hamper UM, Kuhlman JE, Anderson ND, et al. (1995) Single- and double- lumen silicone breast implant integrity: prospective evaluation of MR and US criteria. Radiology 197: 45-52.

4. Baratte A, Bodin F, Del Pin D, Wilk A, Bruant C (2011) [Poland's syndrome in women: Therapeutic indications according to the grade. Apropos of 11 cases and review of the literature]. Ann Chir Plast Esthet 56: $33-42$

5. Ministero della Salute. http://www.salute.gov.it. Accessed 23 September 2012.

6. Dragu A, Theegarten D, Bach AD, Polykandriotis E, Arkudas A, et al. (2009) Intrapulmonary and cutaneous siliconomas after silent silicone breast implant failure. Breast J 15: 496-499. 